\title{
Éclosions de grippe dans les hôpitaux ontariens, 2012 à 2016
}

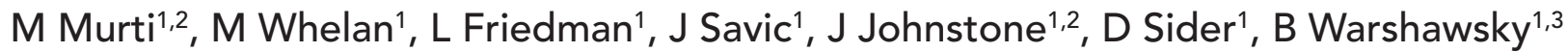

\section{Résumé}

Contexte : Les éclosions de grippe en milieu hospitalier touchent les populations de patients vulnérables et les exposent à un risque considérable de morbidité et de mortalité, mais les données sur ces éclosions sont limitées.

Objectif : Décrire les données de surveillance sur les éclosions de grippe qui ont sévi dans les hôpitaux ontariens entre 2012-13 et 2015-16, et comparer les saisons où le sous-type H3N2 était dominant à celles où le sous-type H1N1 était dominant.

Méthodologie : Les données sur des éclosions de grippe qui ont sévi entre le $1^{\text {er }}$ septembre 2012 et le 31 août 2016 en milieu hospitalier et dont la nature a été confirmée en laboratoire ont été analysées pour y relever des indicateurs de durée et de gravité (taux d'attaque, taux de pneumonie et taux de mortalité). La fréquence, la durée et la gravité des éclosions de grippe de type A ont été comparées entre les saisons où le sous-type H3N2 était dominant (2012-13 et 2014-15) et celles où le sous-type H1N1 était dominant (2013-14 et 2015-16).

Résultats : Au cours des quatre années visées par l'étude, 256 éclosions touchant 1586 patients ont sévi en milieu hospitalier, entraînant 91 cas de pneumonie et 40 décès. La saison de grippe comptant le moins d'éclosions était la saison 2015-16 ( $n=36$ ); celle qui en comptait le plus était la saison 2014-15 ( $n=117)$. La saison 2014-15 est également celle qui comptait le nombre le plus élevé de cas de grippe $(n=753)$, de pneumonie $(n=46)$, de décès $(n=18)$ et d'hôpitaux déclarant au moins une éclosion $(n=72)$. La durée moyenne des éclosions était de 4,5 jours en 2013-14 et de 6,0 jours en 2015-16. Les comparaisons des saisons où les sous-types H3N2 et H1N1 étaient prédominants n'ont pas révélé de différences statistiquement significatives sur le plan de la durée ou de la gravité des éclosions. Par contre, un nombre significativement plus élevé d'éclosions de grippe de type A que d'éclosions de grippe de type B a été déclaré au cours des saisons où le sous-type H3N2 était dominant, comparativement aux saisons où le sous-type H1N1 était dominant $(p<0,05)$.

Conclusion : Bien que les années où le sous-type H3N2 était dominant aient contribué à la morbidité et à la mortalité causées par la grippe en raison de l'augmentation du nombre d'éclosions en milieu hospitalier, aucune différence significative quant à la durée et à la gravité des éclosions de grippe de type A n'a été observée entre les saisons où les sous-types H3N2 et H1N1 étaient dominants.

\author{
Affiliations \\ ${ }^{1}$ Santé publique Ontario, Toronto \\ (Ontario) \\ 2 École de santé publique Dalla \\ Lana, Université de Toronto, \\ Toronto (Ontario) \\ ${ }^{3}$ Université Western, London \\ (Ontario)
}

*Correspondance : michelle. murti@oahpp.ca

Citation proposée : Murti M, Whelan M, Friedman L, Savic J, Johnstone J, Sider D, Warshawsky B. Éclosions de grippe dans les hôpitaux ontariens, 2012 à 2016. Relevé des maladies transmissibles au Canada

2018;44(9):227-32. https://doi.org/10.14745/ccdr.v44i09a03f

Mots-clés : grippe, éclosion, soins actifs, hôpital, morbidité, mortalité

\section{Introduction}

La grippe est une cause importante de morbidité et de mortalité au Canada, où 3500 décès et 12200 hospitalisations attribuables à la grippe saisonnière sont enregistrés chaque année (1). En date du 31 mars 2018, 1663 éclosions de grippe ayant sévi au cours de la saison 2017-18 dans les hôpitaux, les établissements de soins de longue durée (ESLD) et d'autres milieux avaient été déclarées dans les rapports du système Surveillance de l'influenza de l'Agence de la santé publique du Canada (2). Bien que la majorité des éclosions de grippe déclarées à l'échelle nationale aient sévi dans des ESLD, 10,5\% ( $n=175 / 1663$ ) des éclosions sont survenues dans des hôpitaux (2). L'introduction du virus de la grippe en milieu hospitalier par les patients, le personnel et les visiteurs pose problème puisque bon nombre de patients hospitalisés sont vulnérables à la grippe et à ses complications en raison de leur âge, de leur état de santé et de la maladie qui a justifié leur admission. Par exemple, une revue de 12 rapports réalisée en 2002 sur les éclosions de grippe nosocomiales déclarées dans des hôpitaux de soins actifs aux États-Unis a révélé que le taux d'attaque chez les patients atteignait $50 \%$ (distribution : de 3 à $50 \%$ ) et que le taux de mortalité était notable (distribution : de 33 à $60 \%$ ) (3).

Or, au-delà du nombre d'éclosions déclaré dans les rapports du système Surveillance de l'influenza, les données sur les 
caractéristiques des éclosions de grippe dans les hôpitaux canadiens, dont la durée et la gravité, sont très limitées (4). La synthèse des caractéristiques des éclosions d'influenza par les juridictions consolide généralement les données des hôpitaux à celles d'autres établissements tels les ESLD et ce même si ces milieux diffèrent sur le plan des facteurs pouvant avoir une incidence sur la détection, le contrôle et les issues des éclosions (populations de patients, normes de prévention et contrôle des infections, dépistage des virus respiratoires et infrastructures) (5). Comparativement aux ESLD, où la vaste majorité des résidents sont des adultes âgés, les hôpitaux accueillent des patients de tous âges, y compris de jeunes adultes, lesquels ont tendance à être plus vulnérables au sous-type H1N1 du virus de la grippe (6). Chez les adultes âgés, la morbidité et la mortalité sont généralement plus importantes au cours des années où le sous-type H3N2 est dominant (7-10). Par conséquent, les éclosions de grippe en milieu de soins actifs doivent faire l'objet d'une analyse et d'un examen distincts.

Par ailleurs, les caractéristiques des éclosions peuvent varier selon le sous-type du virus en cause. En Ontario, les saisons au cours desquelles le sous-type H3N2 est dominant sont généralement associées à un plus grand nombre d'éclosions de grippe de type A confirmée en laboratoire, comparativement aux saisons où c'est le sous-type H1N1 qui domine. Selon les rapports du système Surveillance de l'influenza, les tendances sont comparables à l'échelle nationale $(4,11)$. Le nombre total d'éclosions est plus élevé au cours des saisons où le sous-type H3N2 domine, soit, mais on ne sait toutefois pas si les éclosions qui sévissent dans les hôpitaux pendant ces saisons sont plus graves sur le plan de la durée et des complications que celles qui sévissent dans les hôpitaux pendant les saisons où c'est le sous-type H1N1 qui domine $(3,12)$. L'objectif du présent rapport est de décrire les éclosions de grippe qui ont sévi dans les hôpitaux ontariens au cours des quatre saisons grippales de I'année 2012-13 à l'année 2015-16, y compris leur fréquence, leur durée et leur gravité, et d'évaluer les différences entre les saisons où les sous-types H3N2 et H1N1 étaient dominants.

\section{Méthodologie}

\section{Cueillette des données}

En Ontario, les ESLD, les maisons de retraite, les hôpitaux et autres établissements doivent déclarer toutes les éclosions de grippe qui sévissent entre leurs murs au bureau de santé publique de leur région (13). Selon la définition donnée par la province, une éclosion de grippe en milieu hospitalier correspond à l'observation d'au moins deux cas d'infection nosocomiale aiguë des voies respiratoires (c'est-à-dire une infection acquise pendant la prestation de soins de santé qui n'était pas présente ou en incubation au moment de l'admission) au cours d'une période de 48 heures dans une unité particulière de l'hôpital. Au moins un des deux cas doit par ailleurs avoir été confirmé en laboratoire $(13,14)$. La zone (p. ex. les unités ou les services) touchée par l'éclosion à l'hôpital est qualifiée de «zone à risque » et comprend tous les patients y étant situés.

L'information sur l'éclosion (p. ex. la date de début et le nombre de patients touchés) est entrée dans le Système intégré d'information sur la santé publique de l'Ontario (SIISP) (13). Les données entrées dans le SIISP comprennent le nombre de cas, les cas avec pneumonie (confirmés par radiographie pulmonaire), les décès liés à l'éclosion et le nombre total de patients dans la zone à risque (13). Le type du virus en cause (type $A$, type $B$, ou les deux) peut être entré dans le SIISP; cependant, le sous-type du virus de type $A(H 3 N 2$ ou H1N1) n'est pas systématiquement inclus

Le 20 mars 2018, nous avons extrait les données suivantes du SIISP : données sur les éclosions déclarées et enrayées qui, selon la description, ont sévi en milieu hospitalier, ou dont le titre comprend le mot « hospital » (hôpital), qui ont sévi entre le $1^{\text {er }}$ septembre 2012 et le 31 août 2016 et qui correspondent à la définition d'éclosion de grippe. Selon la définition, la saison grippale correspond à la période comprise entre le $1^{\text {er }}$ septembre et le 31 août de chaque année. L'éclosion était exclue lorsque le nombre de cas était manquant ou le nom de l'hôpital ne correspondait à aucun des 230 hôpitaux de la liste du ministère de la Santé et des Soins de longue durée de l'Ontario (15). Nous avons inclus les éclosions touchant moins de deux patients dans notre analyse là où des membres du personnel figuraient parmi les personnes touchées (conformément à la définition de l'Ontario).

\section{Analyse des données}

Pour chacune des quatre saisons, nous avons calculé le nombre total de cas associés à l'éclosion de même que les médianes et la distribution de valeurs des résultats suivants : hôpitaux ayant déclaré au moins une éclosion; éclosions par hôpital ayant déclaré une éclosion; durée des éclosions; nombre de cas par éclosion; taux d'attaque (cas par patients hospitalisés dans la zone à risque); nombre de cas de pneumonie; nombre de décès. Les éclosions comportant des données manquantes ou présentant un taux d'attaque supérieur à $100 \%$ ont été exclues des analyses du taux d'attaque et de celles de la durée des éclosions par année.

La définition donnée à la durée d'une éclosion correspondait au nombre de jours compris entre la date de début de l'infection du cas de référence et la date de début de l'infection du dernier cas associé à l'éclosion, conformément aux données entrées dans le SIISP. Cette définition permet l'harmonisation de la méthode de calcul de la durée d'une éclosion à l'autre puisqu'à leur discrétion, ce sont les équipes de gestion des éclosions hospitalières qui déterminent le moment où une éclosion est maîtrisée après la manifestation clinique du dernier cas.

Conformément aux résultats des tests effectués par le laboratoire de Santé publique Ontario, le rapport sommaire annuel du Bulletin sur les pathogènes respiratoires en Ontario comprend la grippe de type dominant, soit la grippe de type $A$, et la proportion de toutes les espèces de sous-types de la grippe de type A (par communauté, hôpital et éclosion) du même sous-type : le sous-type H3N2 était dominant en 2012-13 (90,6\%), le sous-type H1N1 était dominant en 2013-14 $(85,7 \%)$, le sous-type H3N2 était dominant en 2014-15 (99,0\%) et le sous-type H1N1 était dominant en 2015-16 (89,4\%) (11). D'abord, nous avons regroupé des données sur les éclosions de grippe de type $A$ qui ont sévi au cours des années où les sous-types H3N2 et H1N1 étaient dominants. Ensuite, nous avons comparé le nombre d'éclosions et les proportions de cas avec pneumonie et décès entre les saisons où les sous-types H3N2 et H1N1 étaient dominants à l'aide de tests du chi carré. Finalement, nous avons comparé la durée médiane des éclosions, le nombre médian de cas associés aux éclosions et le taux d'attaque médian des saisons où les sous-types H3N2 et H1N1 étaient dominants à l'aide d'un test non paramétrique de Mann-Whitney-Wilcoxon (16-19). Les analyses ont été effectuées à l'aide du logiciel SAS 9.4 (SAS Institute Inc., à Cary, Caroline du Nord, États-Unis). Étant donné que ces analyses étaient 
conformes à la surveillance courante, elles n'ont pas fait l'objet de la revue du Comité d'éthique de Santé publique Ontario.

\section{Résultats}

Entre l'année 2012-13 et l'année 2015-16, 101 hôpitaux ontariens ont déclaré au moins une éclosion de grippe, pour un total de 256 éclosions. Le tableau 1 résume les caractéristiques des éclosions de chaque saison. Les données sur la durée ou le taux d'attaque de 19 des 256 éclosions étaient incomplètes ou dénuées de sens. Ces éclosions ont donc été exclues du rapport sommaire correspondant à l'année de leur éclosion. Une des éclosions comprises dans les éclosions de grippe de type $A$ comportait des cas de grippe de type $A$ et de type $B$.

Tableau 1 : Comparaison des caractéristiques des éclosions de grippe en milieu hospitalier qui ont sévi au cours des saisons 2012-13 à 2015-16 en Ontario

\begin{tabular}{|c|c|c|c|c|}
\hline \multirow{2}{*}{ Résultat } & \multicolumn{4}{|c|}{ Saison grippale } \\
\hline & $2012-13$ & 2013-14 & 2014-15 & $2015-16$ \\
\hline $\begin{array}{l}\text { Nombre total } \\
\text { d'éclosions }\end{array}$ & 65 & 38 & 117 & 36 \\
\hline $\begin{array}{l}\text { - Grippe de type } \\
A(n, \%)\end{array}$ & $63(96,9 \%)$ & $16(42,1 \%)$ & $112(95,7 \%)$ & $27(75,0 \%)$ \\
\hline $\begin{array}{l}\text { - Grippe de type } \\
\text { B (n,\%) }\end{array}$ & $2(3,1 \%)$ & $22(57,9 \%)$ & $5(4,3 \%)$ & $9(25,0 \%)$ \\
\hline $\begin{array}{l}\text { Nombre d'hôpitaux } \\
\text { ayant déclaré } \\
\text { au moins une } \\
\text { éclosion (\% total } \\
\text { d'établissements) }\end{array}$ & $45(19,6 \%)$ & $30(13,0 \%)$ & $72(31,3 \%)$ & $29(12,6 \%)$ \\
\hline $\begin{array}{l}\text { Nombre médian } \\
\text { d'éclosions par } \\
\text { hôpital où une } \\
\text { éclosion a sévi }\end{array}$ & 1,0 & 1,0 & 1,0 & 1,0 \\
\hline - Distribution & $1-6$ & $1-2$ & $1-7$ & $1-4$ \\
\hline $\begin{array}{l}\text { Durée médiane des } \\
\text { éclosions, en jours }\end{array}$ & 5,0 & 4,5 & 5,0 & 6,0 \\
\hline - Distribution & $0-29$ & $1-20$ & $0-32$ & $0-16$ \\
\hline $\begin{array}{l}\text { Nombre total de } \\
\text { cas associés à une } \\
\text { éclosion par saison }\end{array}$ & 465 & 179 & 753 & 189 \\
\hline $\begin{array}{l}\text { Nombre médian de } \\
\text { cas associés à une } \\
\text { éclosion }\end{array}$ & 5,0 & 3,0 & 5,0 & 5,0 \\
\hline - Distribution & $1-45$ & $2-11$ & $2-60$ & $1-14$ \\
\hline $\begin{array}{l}\text { Pourcentage } \\
\text { médian du taux } \\
\text { d'attaque par zone } \\
\text { à risque }\end{array}$ & $16,1 \%$ & $18,5 \%$ & $15,9 \%$ & $18,0 \%$ \\
\hline - Distribution & $4,1-70,8 \%$ & $4,4-75,0 \%$ & $4,1-66,7 \%$ & $4,5-36,8 \%$ \\
\hline $\begin{array}{l}\text { Nombre de cas de } \\
\text { pneumonie (\% de } \\
\text { cas) })^{\mathrm{a}}\end{array}$ & $25(5,4 \%)$ & $15(8,4 \%)$ & $46(6,1 \%)$ & $5(2,6 \%)$ \\
\hline $\begin{array}{l}\text { Nombre de cas } \\
\text { fatals (\% de cas) }\end{array}$ & $12(2,6 \%)$ & $3(1,7 \%)$ & $18(2,4 \%)$ & $7(3,7 \%)$ \\
\hline
\end{tabular}

Selon le nombre total de cas $(n=1586)$

À une éclosion par saison au cours de chacune des quatre années étudiées, le nombre médian d'éclosions par établissement était stable, bien que certains établissements en aient déclaré jusqu'à six (en 2012-13) ou sept (en 2014-15). La durée médiane des éclosions était de 4,5 à 6,0 jours. En tout, 1586 cas de grippe associés à une éclosion ont été déclarés au cours des quatre saisons étudiées. Parmi les personnes atteintes, 91 ont eu une pneumonie $(5,7 \%)$ et 40 sont décédées (2,5\%). Des fluctuations ont été observées sur le plan du pourcentage de personnes qui ont eu une pneumonie et qui sont décédées. Autrement dit, la tendance n'était pas constante d'une saison à l'autre. Sur les quatre saisons étudiées, c'est au cours de la saison 2014-15 que le plus grand nombre d'éclosions $(n=117)$, la proportion la plus importante d'établissements ayant déclaré au moins une éclosion $(31,3 \%)$ et le plus grand nombre de cas cumulatifs associés à une éclosion $(n=753)$ ont été déclarés. C'est également au cours de cette saison que le plus grand nombre d'éclosions en un même établissement ( $n=7)$, les éclosions faisant le plus grand nombre de victimes ( $n=60$ ) et les éclosions les plus longues (32 jours) ont été déclarées.

Le tableau 2 résume les données sur les 218 éclosions de grippe de type A qui ont sévi au cours des saisons où le sous-type H3N2 ( $n=175)$ ou le sous-type H1N1 $(n=43)$ étaient dominants. Au total, 17 éclosions ont été exclues du rapport sommaire des données sur la durée ou de celui sur le taux d'attaque, ou des deux, en raison de données incomplètes ou dénuées de sens. Le nombre d'éclosions de grippe de type A était significativement plus élevé que le nombre d'éclosions de grippe de type $B$ au cours des saisons où le sous-type H3N2 était dominant, comparativement aux saisons où le sous-type H1N1 était dominant $(p<0,05)$. La durée de l'éclosion, le nombre de cas par éclosion, le taux d'attaque, le pourcentage de cas de pneumonie et le pourcentage de cas fatals n'ont pas varié de façon significative selon la souche dominante en circulation.

\section{Tableau 2 : Comparaison des éclosions de grippe de type A dans les hôpitaux au cours des saisons où le sous-type H3N2 ou le sous-type H1N1 étaient dominants de 2012-13 à 2015-16 en Ontario}

\begin{tabular}{|l|r|r|r|}
\hline \multicolumn{1}{|c|}{ Résultat } & \multicolumn{1}{|c|}{$\begin{array}{c}\text { Saisons } \\
\text { H3N2 } \\
\text { (2012-13, } \\
\text { 2014-15) }\end{array}$} & $\begin{array}{r}\text { Saisons } \\
\text { H1N1 } \\
\text { (2013-14, } \\
\text { 2015-16) }\end{array}$ & Valeur p \\
\hline $\begin{array}{l}\text { Nombre d'éclosions de } \\
\text { grippe de type A (\% } \\
\text { d'eclosions de grippe de } \\
\text { type A parmi les eclosions } \\
\text { de grippe de types A et B) }\end{array}$ & 175 (96,2\%) & $43(58,1 \%)$ & $<0,0001^{\text {a }}$ \\
\hline $\begin{array}{l}\text { Durée médiane des éclosions, } \\
\text { en jours }\end{array}$ & 5,0 & 4,0 & $0,56^{\mathrm{b}}$ \\
\hline - Distribution & $0-32$ & $0-16$ & 0 \\
\hline $\begin{array}{l}\text { Nombre médian de cas } \\
\text { associés à une éclosion }\end{array}$ & 5,0 & 5,0 & $0,49^{\mathrm{b}}$ \\
\hline - Distribution & $16,0 \%$ & $18,8 \%$ & 0 \\
\hline $\begin{array}{l}\text { Pourcentage médian du taux } \\
\text { d'attaque par zone à risque } \\
\text { et par éclosion }\end{array}$ & $4,1-70,8 \%$ & $4,5-58,3 \%$ & $0,66^{\mathrm{b}}$ \\
\hline - Distribution & $69(5,9 \%)$ & $14(6,2 \%)$ & $0,85^{\mathrm{a}}$ \\
\hline $\begin{array}{l}\text { Nombre de cas de } \\
\text { pneumonie (\% de cas) }\end{array}$ & $30(2,5 \%)$ & $9(4,0 \%)$ & $0,23^{\mathrm{a}}$ \\
\hline Nombre de décès (\% de cas) & & & 0 \\
\hline
\end{tabular}

Abréviation : $<$, inférieur à

a Test du chi carré

b Test de Mann-Whitney-Wilcoxon

\section{Discussion}

Cette étude a révélé que les éclosions sévissent de façon régulière dans les hôpitaux ontariens et qu'elles contribuent à la morbidité et à la mortalité globales qui sont associées à la grippe. La majorité des hôpitaux n'ont pas déclaré d'éclosions au 
cours des quatre saisons grippales qui ont fait l'objet de l'étude, mais parmi ceux qui en ont déclaré, la médiane saisonnière était d'une éclosion de cinq jours avec cinq patients. Dans une minorité d'établissements, le fardeau de la maladie était élevé. Jusqu'à sept éclosions durant jusqu'à 32 jours et comptant jusqu'à 60 patients ont été déclarées au cours d'une seule saison. Toutes ces éclosions ont sévi au cours de la saison 2014-15, ce qui corrobore d'autres données selon lesquelles le bilan de la saison 2014-15 a été particulièrement lourd en raison de la souche en circulation et de la faible efficacité du vaccin cette année-là (20). Notre comparaison des éclosions de grippe de type $A$ au cours des saisons où les sous-types H3N2 et H1N1 étaient dominants n'a révélé aucune différence significative sur le plan de la durée médiane, du nombre médian de cas, du taux d'attaque, des cas de pneumonie et des cas fatals.

\section{Il s'agit du premier rapport de surveillance qui décrit les} caractéristiques des éclosions qui ont sévi dans les hôpitaux ontariens au cours de plusieurs saisons. Nous n'avons repéré aucun rapport publié comparant les caractéristiques des éclosions de grippe de type $A$ ayant sévi au cours des saisons H3N2 et H1N1. Le peu de données comparables qui ont été publiées par d'autres juridictions suggère qu'au-delà des données sur le nombre total d'éclosions, les rapports de surveillance de la santé publique devraient être utilisés pour comprendre et réduire la morbidité et la mortalité associées aux éclosions.

D'autres études publiées fondées sur la même définition de la durée d'une éclosion que celle que nous avons utilisée dans ces analyses (à savoir le délai entre le premier cas et le dernier cas) ont révélé une durée d'éclosion plus longue que celle que nous avons observée. Dans le cadre d'une revue de 2002 sur les éclosions de grippe dans les hôpitaux de soins actifs, Salgado et ses collaborateurs (3) ont rapporté que la durée médiane des éclosions était de sept jours. Par contre, ces résultats sont fondés sur 12 déclarations d'éclosions provenant d'un éventail d'établissements hospitaliers différents, alors que les nôtres sont fondés sur la surveillance des éclosions qui ont sévi dans les hôpitaux. De plus, dans le cadre d'une revue des éclosions d'affections pseudo-grippales dans les ESLD de Winnipeg, Mahmud et ses collaborateurs (9) ont déclaré que la durée médiane des éclosions était de 16 jours. Le fait que la durée des éclosions était plus longue dans le cadre de la présente étude est peut-être attribuable au milieu (ESLD vs hôpital) et à l'inclusion de cas d'affections pseudo-grippales et non juste des cas de grippe confirmés en laboratoire. Il est à noter que nous avons inclus des éclosions dont tous les cas se sont manifestés au cours de la même journée (autrement dit, des éclosions dont la durée était de zéro jour) dans nos analyses. On ignore si c'est la mise en route rapide des mesures de lutte contre l'éclosion ou la déclaration de données inexactes qui explique que ces éclosions ont duré moins d'une journée.

Les taux d'attaque relevés dans nos analyses sont conformes à la gamme étendue de données déclarées dans les publications sur les éclosions en milieu hospitalier $(3,21)$. Le taux global de décès associés à une éclosion dans le cadre de cette étude $(2,5 \%$; $\mathrm{n}=40$ décès $/ 1586$ cas) est inférieur au taux de mortalité médian de 16 \% dans les hôpitaux de soins actifs et gériatriques déclaré dans la revue de Salgado et ses collaborateurs (3), qui portait sur trois déclarations d'éclosions ayant sévi entre 1960 et 1982. La fréquence accrue des éclosions de grippe de type $A$ au cours des années où le sous-type H3N2 était dominant est conforme à celle qui a été déclarée dans les études révélant que l'infection par le virus de sous-type H3N2 est plus courante que l'infection par le virus de sous-type H1N1 chez les personnes âgées hospitalisées $(12,22)$.

La force principale de notre travail est que nous avons analysé et comparé un nombre important d'éclosions ayant sévi au cours de quatre saisons grippales caractérisées par des degrés différents d'activité grippale dans la collectivité et d'efficacité vaccinale $(16-19,23)$. Les limites de nos conclusions découlent de l'utilisation de données de surveillance recueillies de façon systématique. Dans le SIISP, les données sur les éclosions ne comprennent pas toujours l'information sur les sous-types de la grippe de type A. Par conséquent, il est impossible d'effectuer des comparaisons par sous-type. Le sous-type H3N2 peut avoir été à l'origine de certaines éclosions de grippe de type $A$ qui ont sévi au cours des années où le sous-type H1N1 était dominant. Ce phénomène expliquerait les similitudes entre les caractéristiques des éclosions qui ont sévi au cours des saisons où le sous-type H1N1 ou le sous-type H3N2 était dominant. Le manque de données ou la qualité insuffisante de celles-ci, ou les deux, étaient fréquents pour d'autres éléments, ce qui empêchait la comparaison d'autres aspects des éclosions dans les hôpitaux. Les statistiques regroupées sur l'atteinte des membres du personnel et sur la couverture vaccinale du personnel et des patients dans les zones à risque n'étaient pas toutes comparables sur le plan de l'exhaustivité et de l'exactitude. Ces statistiques étaient donc trop peu fiables pour être incluses dans ces analyses.

L'utilisation de données provinciales regroupées sur les éclosions dans le SIISP est elle aussi limitée puisque des éléments de données qui auraient pu expliquer la variation des caractéristiques des éclosions n'ont pas été saisis dans le système. Les données sur chaque cas (p. ex. l'âge du patient, son état de santé sous-jacent et ses symptômes, les épreuves de laboratoire ou l'usage d'antiviraux) n'étaient pas non plus disponibles, ce qui a empêché l'évaluation de leur incidence sur la gravité d'une éclosion. En outre, l'information sur le risque de transmission dans la zone touchée par l'éclosion (p. ex. l'acuité ou le type d'unités de soins dans l'hôpital, I'âge de l'hôpital, la configuration de la chambre ou de l'unité de soins, la quantité de ressources de prévention et contrôle des infections de l'hôpital) n'est pas déclarée.

Plusieurs zones grises sont associées à ces données. Par exemple, le fait que certains hôpitaux n'aient pas déclaré d'éclosions, alors que d'autres en ont déclaré un nombre élevé, s'explique peut-être par la variation des pratiques de prévention et de contrôle des infections et des méthodes de déclaration des éclosions. Dans les services de soins actifs, où le roulement de patients est supérieur, il est plus probable qu'une éclosion ne soit pas reconnue, car des patients infectés pourraient obtenir leur congé avant la détection d'une grippe nosocomiale. En revanche, la durée des séjours dans les établissements de soins chroniques ou complexes est plus longue, ce qui augmente non seulement le risque de transmission de la grippe, mais également la probabilité de la détection de l'infection. L'interprétation de la méthode de déclaration de l'information sur les éclosions peut varier d'un hôpital et d'un bureau de santé publique à l'autre, résultant en une variabilité des valeurs déclarées. Par exemple, pour certaines éclosions, le nombre de patients déclaré dans la zone à risque est le même que le nombre total de patients dans l'hôpital. Il est impossible de savoir si ces éclosions ont touché I'hôpital en entier ou si les données déclarées sont inexactes. Les différences d'interprétation du dénominateur utilisé pour calculer le nombre de patients dans la zone à risque (lits disponibles vs nombre total de patients présents à n'importe quel moment 
pendant l'éclosion) peuvent elles aussi influencer le taux d'attaque.

Ces analyses portent précisément sur l'Ontario, et les caractéristiques des éclosions sont influencées par les lignes directrices provinciales sur la gestion des éclosions de grippe dans les hôpitaux ainsi que les pratiques de déclaration (24). L'étude des éclosions hospitalières en provenance $d$ 'autres provinces et juridictions permettrait d'effectuer une comparaison entre leurs données et celles sur l'Ontario afin d'établir des cibles de santé publique visant la réduction de la morbidité et de la mortalité associées aux éclosions de grippe en milieu hospitalier. L'efficacité très faible du vaccin de la saison 2014-15 (23) ainsi que d'autres variations observées d'une saison grippale à l'autre justifient l'inclusion de plusieurs saisons dans les futures études pour mieux caractériser la diversité des éclosions qui sévissent dans les hôpitaux.

\section{Conclusion}

Les éclosions de grippe sont courantes en milieu hospitalier et contribuent à la morbidité et à la mortalité associées à cette maladie. En somme, nous avons conclu qu'un bon nombre de caractéristiques d'éclosions de grippe en milieu hospitalier, dont la durée médiane, le nombre médian de cas par éclosion et le taux d'attaque, étaient plutôt semblables au cours des quatre saisons grippales étudiées. Cette similitude ne dépendait pas du sous-type dominant de la grippe de type $A$, même si un plus grand nombre d'éclosions de grippe de type $A$ ont été déclarées au cours des saisons où le sous-type H3N2 était dominant qu'au cours des saisons où le sous-type H1N1 était dominant. Une amélioration de l'exhaustivité, de l'exactitude et de la cohérence des statistiques sommaires sur les éclosions tirées des rapports de surveillance de la santé publique permettrait de faire des analyses plus rigoureuses. Une réflexion plus approfondie s'impose également pour déterminer les données minimales requises sur les cas, les éclosions et les hôpitaux afin que les rapports de données agrégées permettent de répondre aux questions de santé publique sur la surveillance, la gestion et l'évaluation des éclosions de grippe en milieu hospitalier.

\section{Déclaration des auteurs}

M. M. - Conceptualisation, rédaction - ébauche originale, rédaction - examen et révision, visualisation

M. W. - Analyses officielles, rédaction - sections de l'ébauche originale, rédaction - examen et révision, visualisation

L. F. - Rédaction - sections de l'ébauche originale, rédaction examen et révision

J. S. - Analyses officielles, rédaction - examen et révision

J. J. - Rédaction - examen et révision

D. S. - Rédaction - examen et révision

B. W. - Rédaction - examen et révision

\section{Conflit d'intérêts}

Aucun.

\section{Remerciements}

Les auteurs souhaitent remercier les membres du personnel des hôpitaux et des bureaux de santé publique qui ont participé à la gestion et à la déclaration de ces éclosions.
Les auteurs souhaitent également remercier Shelly Bolotin et Karin Hohenadal pour leur aide à la révision de ce travail.

\section{Financement}

Ce travail a été réalisé grâce au soutien de Santé publique Ontario.

\section{Références}

1. Schanzer DL, Sevenhuysen C, Winchester B, Mersereau T. Estimating influenza deaths in Canada, 1992-2009. PLoS One 2013 Nov;8(11):e80481. https://doi.org/10.1371/ journal.pone.0080481. PubMed (https://www.ncbi.nlm. nih.gov/entrez/query.fcgi?cmd=Retrieve \&db=PubMed\&lis t_uids=24312225\&dopt=Abstract)

2. Agence de la santé publique du Canada. Surveillance de l'influenza: Du 25 mars au 31 mars 2018 (semaine de déclaration 13). Ottawa, ON: Gouvernement du Canada; 2018. https://www.canada.ca/fr/sante-publique/ services/publications/maladies-et-affections/surveillanceinfluenza/2017-2018/semaine13-25-mars-31-mars-2018.html

3. Salgado CD, Farr BM, Hall KK, Hayden FG. Influenza in the acute hospital setting. Lancet Infect Dis 2002 Mar;2(3):145-55. https://doi.org/10.1016/S1473-3099(02)00221-9. PubMed (https://www.ncbi.nlm.nih.gov/entrez/query.fcgi?cmd=Retrieve $\& d b=$ PubMed\&list_uids=11944184\&dopt=Abstract)

4. Agence de la santé publique du Canada. À propos de Surveillance de l'influenza. Ottawa, ON: ASPC; 2018. https:// www.canada.ca/fr/sante-publique/services/maladies/grippeinfluenza/surveillance-influenza/propos-surveillance-influenza. html

5. Medical Officer of Health, Toronto Public Health. 2015 Influenza Update. Toronto, ON: City of Toronto; 2015. https://www.toronto.ca/legdocs/mmis/2015/hl/bgrd/ backgroundfile-85948.pdf

6. Organisation mondiale de la Santé, Bureau Régionale de l'Europe. Selon l'évaluation des risques de la saison grippale 2015-2016, A(H1N1) circule comme virus saisonnier, mais est inclus dans le vaccin. Copenhagen, Danemark: OMS/Europe; 2016. http://www.euro.who.int/fr/health-topics/communicablediseases/influenza/news/news/2016/02/risk-assessment-of-the20152016-influenza-season-confirms-that-ah1n1-is-circulatingas-a-seasonal-virus-but-is-included-in-the-vaccine

7. Kaji M, Watanabe A, Aizawa H. Differences in clinical features between influenza A H1N1, A H3N2, and B in adult patients. Respirology 2003 Jun;8(2):231-3. https://doi.org/10.1046/ j.1440-1843.2003.00457.x. PubMed (https://www.ncbi.nlm.nih. gov/pubmed/12753540)

8. Lee BE, Mukhi SN, Drews SJ. Association between patient age and influenza A subtype during influenza outbreaks. Infect Control Hosp Epidemiol 2010 May;31(5):535-7. https:// doi.org/10.1086/652159. PubMed (https://www.ncbi.nlm. nih.gov/entrez/query.fcgi?cmd=Retrieve $\& d b=$ PubMed\&lis t_uids=12753540\&dopt=Abstract)

9. Mahmud SM, Thompson LH, Nowicki DL, Plourde PJ. Outbreaks of influenza-like illness in long-term care facilities in Winnipeg, Canada. Influenza Other Respir Viruses 2013 
Nov;7(6):1055-61. https://doi.org/10.1111/irv.12052. PubMed (https://www.ncbi.nlm.nih.gov/entrez/query.fcgi?cmd=Retrieve $\& d b=$ PubMed\&list_uids=23145997\&dopt=Abstract)

10. Thompson WW, Shay DK, Weintraub E, Brammer L, Cox N, Anderson LJ, Fukuda K. Mortality associated with influenza and respiratory syncytial virus in the United States. JAMA 2003 Jan;289(2):179-86. https://doi.org/10.1001/jama.289.2.179. PubMed (https://www.ncbi.nlm.nih.gov/entrez/query.fcgi?cmd $=$ Retrieve $\& \mathrm{db}=$ PubMed\&list_uids=12517228\&dopt=Abstract)

11. Ontario Agency for Health Protection and Promotion (Public Health Ontario). Ontario Respiratory Pathogen Bulletin. Toronto, ON: Queenıs Printer for Ontario; 2018. www.publichealthontario.ca/en/ServicesAndTools/ SurveillanceServices/Pages/Ontario-Respiratory-Virus-Bulletin. aspx

12. Toronto Invasive Bacterial Diseases Network. Surveillance de la grippe confirmée en laboratoire nécessitant une hospitalisation chez les adultes, région métropolitaine de Toronto/Peel, saison grippale 2004-2005. Relevé des maladies transmissibles au Canada 2005 Déc;31(24):249-55. PubMed (https://www. ncbi.nlm.nih.gov/entrez/query.fcgi?cmd=Retrieve \&db=PubMe d\&list_uids=16669120\&dopt=Abstract)

13. Ontario Agency for Health Protection and Promotion (Public Health Ontario). iPHIS user guide: Respiratory infection outbreaks in institutions. User Guide v. 1.0. Toronto, ON: Queenss Printer for Ontario; 2014.

14. Ministry of Health and Long-Term Care. Ontario Public Health Standards, Appendix B: Provincial Case Definition for Reportable Diseases: Influenza. Toronto, ON: Queenıs Printer for Ontario; 2014.

15. Clostridium difficile Infection in Ontario Hospitals, January 2009 to December 2013. 2013. http://www.hqontario.ca/ portals/0/documents/system-performance/clostridium-difficileinfection.xls

16. Ontario Agency for Health Protection and Promotion (Public Health Ontario). Ontario Respiratory Virus Bulletin, 201415: Surveillance Season (September 1, 2014 - August 31, 2015). Toronto, ON: Queenss Printer for Ontario; 2015. www. publichealthontario.ca/en/DataAndAnalytics/Documents/ Ontario_Respiratory_Virus_Bulletin-2014-2015_Season_ Summary.pdf

17. Ontario Agency for Health Protection and Promotion (Public Health Ontario). Influenza and respiratory infection surveillance summary report: 2012-13 season. Toronto, ON: Queenıs Printer for Ontario; 2015. www.publichealthontario.ca/en/
eRepository/Influenza_Respiratory_Infection_Surveillance_ Summary_Report_2012_13.pdf

18. Ontario Respiratory Pathogen Bulletin. 2015-16: Surveillance Season (September 1, 2015 - August 31, 2016) Toronto, ON: Queen ss Printer for Ontario. 2016. www. publichealthontario. ca/en/DataAndAnalytics/Documents/Ontario_Respiratory_ Pathogen_Bulletin-Season_Summary_-_2015-16.pdf

19. Ontario Agency for Health Protection and Promotion (Public Health Ontario). Ontario Respiratory Virus Bulletin, 20132014: Surveillance Season (September 1, 2013 -August 31, 2014). Toronto, ON: Queenss Printer for Ontario; 2014. www. publichealthontario.ca/en/DataAndAnalytics/Documents/ Ontario_Respiratory_Virus_Bulletin-2013-2014_Season_ Summary.pdf

20. Skowronski DM, Chambers C, Sabaiduc S, De Serres G, Winter AL, Dickinson JA, Krajden M, Gubbay JB, Drews SJ, Martineau C, Eshaghi A, Kwindt TL, Bastien N, Li Y. A Perfect Storm: Impact of Genomic Variation and Serial Vaccination on Low Influenza Vaccine Effectiveness During the 20142015 Season. Clin Infect Dis 2016 Jul;63(1):21-32. https:// doi.org/10.1093/cid/ciw176. PubMed (https://www.ncbi.nlm. nih.gov/entrez/query.fcgi?cmd=Retrieve\&db=PubMed\&lis t_uids=27025838\&dopt=Abstract)

21. Voirin N, Barret $B$, Metzger MH, Vanhems P. Hospital-acquired influenza: a synthesis using the Outbreak Reports and Intervention Studies of Nosocomial Infection (ORION) statement. J Hosp Infect 2009 Jan;71(1):1-14. https://doi. org/10.1016/j.jhin.2008.08.013. PubMed (https://www.ncbi. $\mathrm{nlm}$.nih.gov/entrez/query.fcgi?cmd=Retrieve\&db=PubMed\&li st_uids=18952319\&dopt=Abstract)

22. Rebbapragada A, Perusini SJ, Blair J, Drews S, Green KA, Plevneshi A, Low DE, McGeer A Epidemiology of severe infection due to influenza $\mathrm{A}(\mathrm{H} 1 \mathrm{~N} 1)$ and $\mathrm{A}(\mathrm{H} 3 \mathrm{~N} 2)$ : Are there differences? 49th Annual Interscience Conference on Antimicrobial Agents and Chemotherapy; 11-15 September 2009; San Francisco, CA.

23. Canadian Influenza Sentinel Practitioner Surveillance Network. SPSN influenza vaccine effectiveness estimates \% $(95 \% \mathrm{Cl})$, 2004-05 to 20016-17 seasons. Vancouver, BC: Canadian SPSN; 2017. www.bccdc.ca/resource-gallery/Documents/ Statistics\%20and\%20Research/Publications/Epid/Influenza\%20 and\%20Respiratory/SPSN_VE_By_Year_Table_2017-12-01.pdf

24. Ontario Hospital Association, Ontario Medical Association. Influenza Surveillance Protocol for Ontario Hospitals. Toronto, ON: Ontario Hospital Association; 2017. www.oha.com/ Documents/Influenza\%20Protocol\%20Revised\%20May\%20 2017.pdf 